\title{
Scales of Mass Generation for Quarks, Leptons and Majorana Neutrinos
}

\author{
Duane A. Dicus and Hong-Jian He \\ Center for Particle Physics, University of Texas at Austin, TX 78712, USA
}

\begin{abstract}
We study $2 \rightarrow n$ inelastic fermion-(anti)fermion scattering into multiple longitudinal weak gauge bosons and derive universal upper bounds on the scales of fermion mass generation by imposing unitarity of the $S$-matrix. We place new upper limits on the scales of fermion mass generation, independent of the electroweak symmetry breaking scale. We find that the strongest $2 \rightarrow n$ limits fall in a narrow range, $3-170 \mathrm{TeV}$ (with $n=2-24$ ), depending on the observed fermion masses.
\end{abstract}

PACS numbers: 12.15.Ff, 14.60.Pq, 11.80.-m, 12.60.-i

Phys. Rev. Lett. (2005), in Press.

\section{New Puzzle for Scales of Fermion Mass Generation}

Understanding scales of mass generation for quarks, leptons and Majorana neutrinos poses a great challenge in elementary particle physics. Mass generation in the standard model (SM) relies on a single hypothetical Higgs boson. Although the masses of weak bosons $\left(W^{ \pm}, Z^{0}\right)$ only involve electroweak gauge couplings $\left(g, g^{\prime}\right)$ and the vacuum expectation value $v=\left(\sqrt{2} G_{F}\right)^{-\frac{1}{2}}$, the quark/lepton masses arise from products of Yukawa couplings $y_{f}$ and $v$. Contrary to the gauge interactions, the Yukawa couplings $y_{f}$ are flavor-dependent and completely arbitrary, exhibiting a huge empirical hierarchy $y_{e} / y_{t}=m_{e} / m_{t} \simeq 3 \times 10^{-6}$ between the electron and the top quark. No compelling principle requires the fermion mass generation to share the same mechanism as the $W / Z$ gauge bosons. Furthermore, the tiny neutrino masses $0.05 \mathrm{eV} \lesssim m_{\nu} \lesssim 1 \mathrm{eV}[1]$ cannot be generated by such a Higgs boson without losing renormalizability [2] or extending the SM particle spectrum 3 , 4]. So far, no Higgs boson has been found, nor is any Yukawa coupling experimentally measured - the origin and scale of fermion mass generation remain completely unknown.

What is wrong with just putting all the bare masses into the SM Lagrangian by hand? Such bare massterms can be made gauge-invariant in the nonlinear realization [5], but are manifestly nonrenormalizable. This causes unitarity violation in high energy scattering at a scale $E^{\star}$. Generically, we define the scale $\Lambda_{x}$ for generating a mass $m_{x}$ to be the minimal energy above which the bare mass term for $m_{x}$ has to be replaced by a renormalizable interaction (adding at least one new physical state to the particle spectrum). Hence, the unitarity violation scale $E^{\star}$ puts a universal upper limit on $\Lambda_{x}, \Lambda_{x} \leqslant E^{\star}$.

A bare mass-term for $\left(W^{ \pm}, Z^{0}\right)$ will cause the high energy $2 \rightarrow 2$ scattering of longitudinal weak bosons to violate unitarity at a scale [6], $E_{W}^{\star} \simeq \sqrt{8 \pi} v \simeq 1.2 \mathrm{TeV}$. This puts an upper limit on the scale of electroweak symmetry breaking (EWSB) and justifies the TeV energy scale for the Large Hadron Collider (LHC) at CERN. Similarly, by adding bare mass terms for Dirac fermions an upper bound on the scale of fermion mass generation can be derived from the $2 \rightarrow 2$ inelastic scattering $f \bar{f} \rightarrow V_{L}^{a_{1}} V_{L}^{a_{2}}\left(V^{a}=W^{ \pm}, Z^{0}\right)$ [], [8],

$$
E_{f}^{\star} \simeq \frac{8 \pi v^{2}}{\sqrt{N_{c}} m_{f}}
$$

where $N_{c}=3$ (1) for quarks (leptons). Since this limit is proportional to $1 / m_{f}$, it is independent of the bound $E_{W}^{\star}$ on the EWSB scale. For all the SM fermions (except the top quark), the limit (10) is substantially higher than $E_{W}^{\star}$. For the scale of mass generation for Majorana neutrinos, Refs. [9, 10] derived an analogous $2 \rightarrow 2$ bound from the scattering $\nu_{L} \nu_{L} \rightarrow V_{L}^{a_{1}} V_{L}^{a_{2}}$,

$$
E_{\nu}^{\star} \simeq 4 \pi v^{2} / m_{\nu}
$$

which is around $10^{16} \mathrm{GeV}$ (the GUT scale) for a typical neutrino mass $m_{\nu} \sim 0.05 \mathrm{eV}[1]$.

However, for $2 \rightarrow n$ inelastic scattering, $f \bar{f}, \nu_{L} \nu_{L} \rightarrow$ $n V_{L}^{a}(n \geqslant 3)$, Ref. [10] found that the $n$-body phase space integration contributes a large energy factor, $s^{n-2}$, to further enhance the cross section (in addition to the energy dependence $s^{1}$ from the squared amplitude), where $\sqrt{s}$ is the center of mass energy. From the unitarity condition on the total cross section [10],

$$
\sigma_{\text {inel }}[2 \rightarrow n] \leqslant 4 \pi / s,
$$

the unitarity limit for $f\left(\nu_{L}\right)$ would behave as 10 .

$$
E_{f, \nu}^{\star} \sim v\left(v / m_{f, \nu}\right)^{\frac{1}{n-1}} \longrightarrow v, \quad(\text { as } n \rightarrow \text { large }),
$$

which could be pushed arbitrarily close to the weak scale $v$ and thus become independent of the fermion mass $m_{f, \nu}$ for large enough $n$. This raises a striking question: is there an independent new scale for fermion mass generation revealed from the fermion-(anti)fermion scattering into weak bosons? We find the behavior (4) very counterintuitive since the kinematic condition forces any $2 \rightarrow n$ unitarity limit $E^{\star}$ to grow at least linearly with $n$,

$$
\sqrt{s}>n M_{W(Z)} \simeq \frac{n}{3} v, \quad \Longrightarrow \frac{E^{\star}}{v}>\frac{n}{3},
$$

which contradicts (4), posing a new puzzle.

Resolution of the New Puzzle

Consider high energy $2 \rightarrow n$ inelastic scattering $f \bar{f}$, $\nu_{L} \nu_{L} \rightarrow n V_{L}^{a}$. Its amplitude can be derived from the corresponding scattering $f \bar{f}, \nu_{L} \nu_{L} \rightarrow n \pi^{a}$ with multiple Goldstone bosons $\left(\pi^{a}\right)$ via the equivalence theorem [11]. In the nonlinear realization, the bare massterms for quarks/leptons and Majorana neutrinos result 


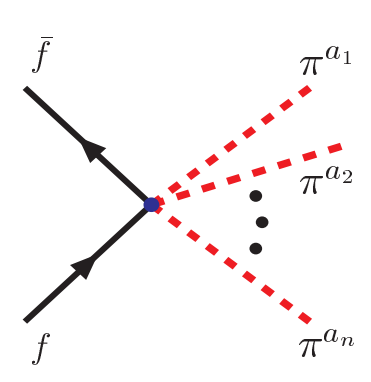

$\mathcal{O}\left(E^{1}\right)$

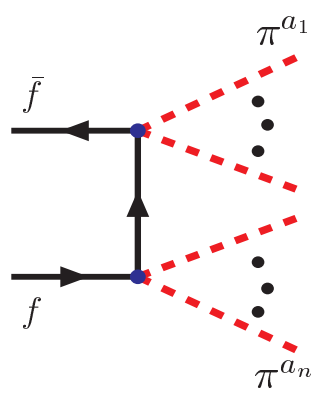

$\mathcal{O}\left(E^{0}\right)$

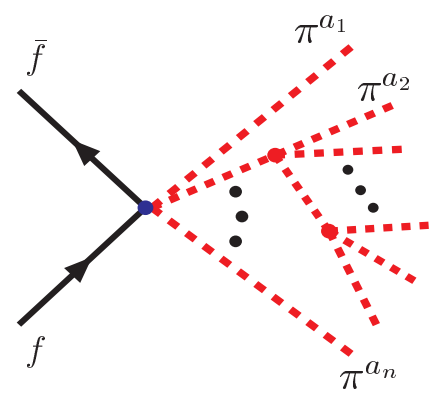

$\mathcal{O}\left(E^{1}\right)$

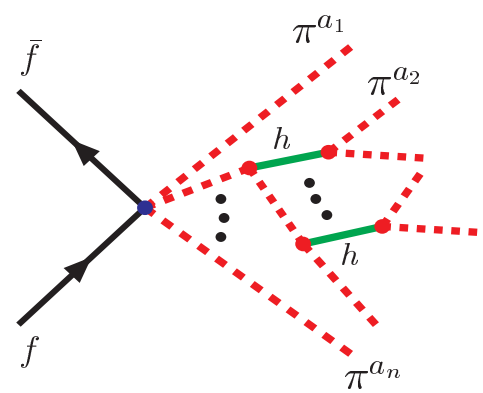

$\mathcal{O}\left(E^{1}\right)$

(d)

(a)

(b)

(c)

FIG. 1: Typical contributions to $f \bar{f}\left(\nu_{L} \nu_{L}\right) \rightarrow n \pi^{a}$ scattering: (a) leading "contact" diagrams of $\mathcal{O}\left(E^{1}\right)$; (b) sub-leading " $t(u)$ channel" diagrams of $\mathcal{O}\left(E^{0}\right)$ which can be ignored for the unitarity analysis; (c) "hybrid" contact diagrams $(n \geqslant 3)$ including Goldstone self-interactions from the EWSB sector; (d) "hybrid" contact diagrams $(n \geqslant 3)$ including Goldstone interactions with the EWSB quanta (illustrated here with the SM Higgs boson $h^{0}$ as the simplest example of EWSB).

in contact interactions between these fermions and Goldstones, of the type $f-\bar{f}-\pi^{n}$ and $\nu_{L^{-}} \nu_{L^{-}} \pi^{n}(n \geqslant 1)$ whose couplings are proportional to the corresponding fermion mass $m_{f(\nu)}$. We classify the relevant contributions in Fig. 1. The contact diagram Fig. 1(a) gives a universal leading amplitude of $\mathcal{O}\left(m_{f, \nu} E / v^{n}\right)$ according to power counting [12], while the non-contact diagrams in Fig. 1(b) are all subleading in energy. The contributions in Fig. 1(c,d) involve the Goldstone self-interactions and the new particle(s) generating the EWSB (illustrated here by the SM Higgs boson $h^{0}$ ) which are model-dependent. A quantitative analysis of Fig. 1(a,d) showed 13 that they may enhance the unitarity bound by a factor of $[\mathcal{O}(2-3)]^{\frac{1}{n-1}}(n \geqslant 3)$ which is very close to one for large $n$. Thus it is justified to use the universal Fig. 1(a) as a model-independent, conservative estimate for the unitarity bound.

Since power counting shows the leading amplitude of $2 \rightarrow n$ scattering to be $\mathcal{O}(1)\left(m_{f, \nu}\right)^{2-\delta} s^{\delta / 2} / v^{n}$ for $n \geqslant 2$, we deduce the generical form of the squared amplitude,

$$
|\mathcal{T}|^{2}=c_{0}\left(\theta_{j}\right)\left(2 N_{c}\right)^{2-\delta} m_{f, \nu}^{2(2-\delta)} s^{\delta} / v^{2 n},
$$

where $\delta=1(2)$ for $f \bar{f}, \nu_{L} \nu_{L},\left(V_{L}^{a_{1}} V_{L}^{a_{2}}\right) \rightarrow n V_{L}^{a}$ in the spin/color-singlet channel, and $c_{0}\left(\theta_{j}\right)$ is a dimensionless coefficient with possible angular dependence. Using (6) we will compute the corresponding total cross section $\sigma_{\text {inel }}[2 \rightarrow n]$ and determine its unitarity violation scale from the condition (3).

We observe that the key for resolving the puzzle posed in Eqs. (44) and (5) is to analyze the additional $n$-dependent dimensionless factors in the exact n-body phase space integration; these factors will sufficiently suppress the $E$-power enhancement $s^{n-2}$ in Eq. (4). Using (6), and including the exact $n$-body phase space for the cross section computation, we can formally derive the $2 \rightarrow n$ unitarity limit,

$$
\begin{gathered}
E^{\star}=v\left[C_{0} 2^{4 n-2} \pi^{2(n-1)} \varrho(n-1) !(n-2) !\left(2 N_{c}\right)^{\delta-2}\right. \\
\left.\times\left(v / m_{f, \nu}\right)^{2(2-\delta)}\right]^{\frac{1}{2(n-2+\delta)}}
\end{gathered}
$$

where $\varrho$ is a symmetry factor for identical particles in the final state. The factor $C_{0}$ originates from the coefficient $c_{0}\left(\theta_{j}\right)$ whose possible angular dependence is included in the exact $n$-body phase space integration. For the leading contact contributions in Fig. 1(a) no such angular dependence exists. Since all large $n$ factors are explicitly counted in (7), we expect that, independent of detail, $C_{0}^{\frac{1}{2(n-2+\delta)}} \sim 1$ becomes increasingly more accurate as $n$ gets larger. Thus, setting $\left(C_{0} \varrho\right)^{1 / 2(n-2+\delta)} \approx 1$ in (7) for simplicity, we derive an estimated unitarity limit $E^{\star}$ which is shown in Fig. 2 (where $m_{\nu}=0.05 \mathrm{eV}$ is chosen). Using the Stirling formula $n ! \simeq n^{n} e^{-n} \sqrt{2 \pi n}$, we deduce the correct asymptotic behavior from (7),

$$
E^{\star} \rightarrow v \frac{4 \pi n}{e}>v \frac{n}{3}, \quad(\text { for } n \gg 1),
$$

which agrees with our kinematic condition (5).

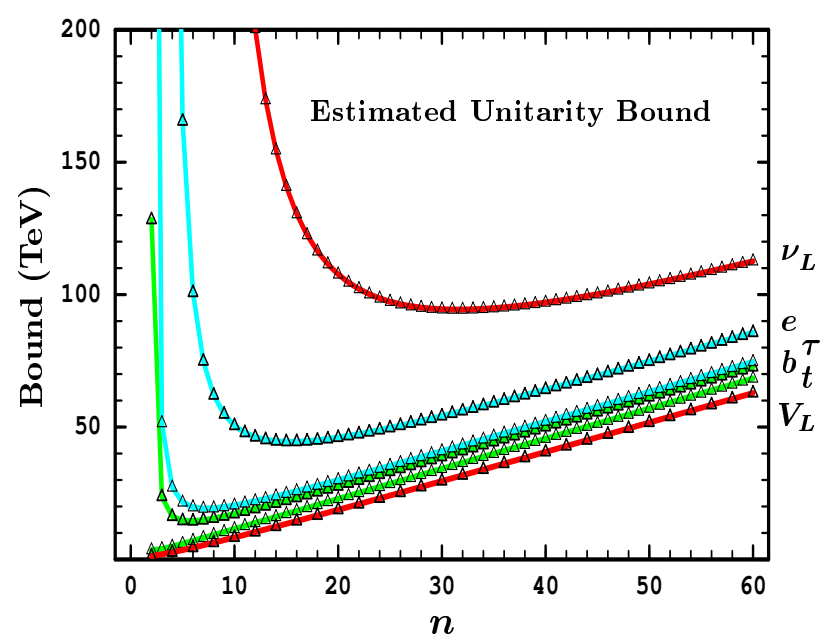

FIG. 2: Realistic estimates of the unitarity limit $E^{\star}$, from the scatterings $V_{L}^{a_{1}} V_{L}^{a_{2}}, t \bar{t}, b \bar{b}, \tau^{-} \tau^{+}, e^{-} e^{+}, \nu_{L} \nu_{L} \rightarrow n V_{L}^{a}$ (curves from bottom to top), as a function of integer $n(\geqslant 2)$.

Fig. 2 shows that for $V_{L}^{a_{1}} V_{L}^{a_{2}}$ and $t \bar{t}$ initial states the strongest bound (minimum of the curve) still occurs at 
$n=n_{s}=2$; while for all light fermions including Majorana neutrinos the best limit for the scale of mass generation lies at a new minimum with $n=n_{s}>2$ and $E^{\star}$ no higher than about $100 \mathrm{TeV}$. These limits are substantially tighter than the corresponding classic $2 \rightarrow 2$ bounds. The more precise calculations below will show these estimates are accurate to within a factor of two. We stress that it is the competition between the large asymptotic linear growth (8) and the strong power suppression (4) that generates a genuine new minimum scale $E_{\text {min }}^{\star}$ for all light fermions at $n=n_{s}>2$, independent of the EWSB scale.

\section{$\underline{\text { Scales of Mass Generation for Quarks and Leptons }}$}

To improve the estimates in Fig. 2, we will precisely compute the dimensionless coefficient $c_{0}$ in the leading squared-amplitude (6), so we can determine the constant $C_{0}$ in Eq. (7) for each given $f \bar{f}, \nu_{L} \nu_{L} \rightarrow n V_{L}^{a}$ process (where the possible identical particle factor $\varrho$ will be explicitly included). We first consider a pair of SM Dirac fermions $\left(f, f^{\prime}\right)$ which form a left-handed $S U(2)_{L}$ doublet $F_{L}=\left(f_{L}, f_{L}^{\prime}\right)^{T}$ and two right-handed weak singlets $f_{R}$ and $f_{R}^{\prime}$. We can formulate their bare Dirac massterms $-m_{f} \bar{f} f-m_{f^{\prime}} \overline{f^{\prime}} f^{\prime}$ into the gauge-invariant nonlinear form,

$$
\mathcal{L}_{f}=-m_{f} \overline{F_{L}} U\left(\begin{array}{l}
1 \\
0
\end{array}\right) f_{R}-m_{f}, \overline{F_{L}} U\left(\begin{array}{l}
0 \\
1
\end{array}\right) f_{R}^{\prime}+\text { H.c. }
$$

where $U=\exp \left[i \pi^{a} \tau^{a} / v\right]$. This gives $f-\bar{f}-\pi^{n}(n \geqslant 1)$ contact interactions between fermions and Goldstone bosons. We compute the leading scattering amplitude for $\mid$ in $\rangle \rightarrow\left(\pi^{+}\right)^{k}\left(\pi^{-}\right)^{\ell}\left(\pi^{0}\right)^{n-k-\ell}$, where the initial state $\mid$ in $\rangle$ consists of two fermions in the color-singlet channel [13].

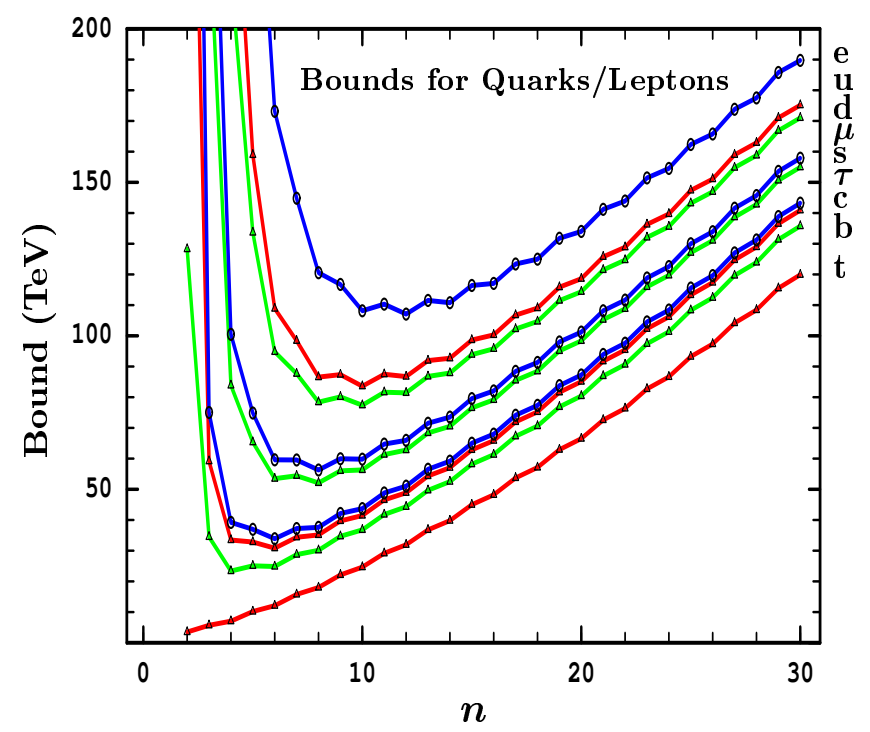

FIG. 3: Precise unitarity bounds $E_{f}^{\star}$ from scatterings $\xi_{1} \xi_{2} \rightarrow$ $n \pi^{a}\left(n V_{L}^{a}\right)$ as a function of $n$, where $\xi_{1} \xi_{2}=t \bar{t}, b \bar{b}, c \bar{c}$, $\tau^{-} \tau^{+}, s \bar{s}, \mu^{-} \mu^{+}, d \bar{d}, u \bar{u}, e^{-} e^{+}$for $n=$ even and $\xi_{1} \xi_{2}=$ $t_{+} \bar{b}_{+}, t_{-} \bar{b}_{-}, c_{+} \bar{s}_{+}, \tau_{-}^{+} \nu_{-}, c_{-} \bar{s}_{-}, \mu_{-}^{+} \nu_{-}, u_{-} \bar{d}_{-}, u_{+} \bar{d}_{+}, e_{-}^{+} \nu_{-}$ for $n=$ odd, (curves from bottom to top). The subscripts \pm refer to helicity.
Imposing unitarity on the $s$-partial wave, we derive corresponding unitarity bound for quarks and leptons,

$$
E_{f}^{\star}=v\left[\left(\frac{v}{m_{\widehat{f}}}\right)^{2} \frac{4 \pi}{N_{c} \widehat{\mathcal{R}}_{k}^{\max }}\right]^{\frac{1}{2(n-1)}},
$$

where $\widehat{f} \in\left(f, f^{\prime}\right)$, and $\widehat{\mathcal{R}}_{k}^{\max } \in\left(2 \mathcal{R}_{1}^{\max }, \mathcal{R}_{2}^{\max }\right)$ with

$$
\begin{aligned}
& \mathcal{R}_{1}^{\max }=\frac{\left(\frac{n}{2} !\right)^{2}}{2^{3 n-4} \pi^{2 n-3}(n !)^{2}(n-1) !(n-2) !}, \\
& \mathcal{R}_{2}^{\max }=\frac{\left(\frac{n-1}{2}\right) !\left(\frac{n+1}{2}\right) !}{2^{3 n-4} \pi^{2 n-3}(n !)^{2}(n-1) !(n-2) !},
\end{aligned}
$$

for $n=$ (even, odd), respectively. We depict the bound $E_{f}^{\star}$ in Fig. 3, which shows that the best limits for all light fermions occur at a new minimum $n=n_{s}>2$ with $E_{b}^{\star}=24.5 \mathrm{TeV}$ for $b$ quark and $E_{e}^{\star}=107 \mathrm{TeV}$ for electron. These two limits only differ by a factor of $\sim 4$.

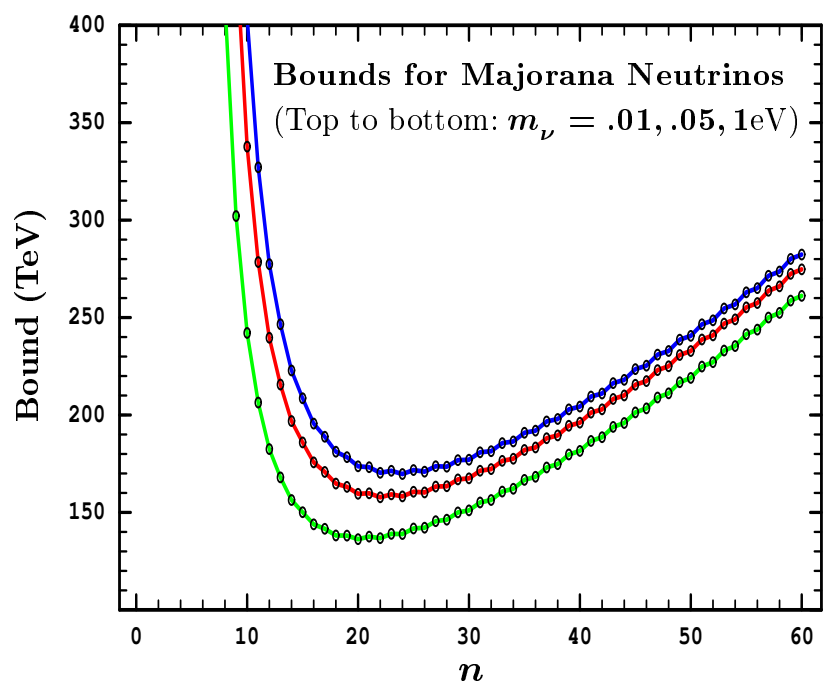

FIG. 4: Scales of mass generation for Majorana neutrinos: the precise unitarity bounds $E_{\nu}^{\star}$ are plotted as a function of $n(\geqslant 2)$. Only integer values of $n$ have a physical meaning.

\section{Scales of Mass Generation for Majorana Neutrinos}

For Majorana neutrinos, we write their dimension-3 bare mass term as $-\frac{1}{2} m_{\nu}^{i j} \nu_{L i}^{T} \widehat{C} \nu_{L j}+$ H.c., which takes the gauge-invariant nonlinear form

$$
\mathcal{L}_{\nu}=-\frac{1}{2} m_{\nu}^{i j} L_{i}^{\alpha T} \widehat{C} L_{j}^{\beta} \bar{\Phi}^{\alpha^{\prime}} \bar{\Phi}^{\beta^{\prime}} \epsilon^{\alpha \alpha^{\prime}} \epsilon^{\beta \beta^{\prime}}+\text { H.c. },
$$

where $\widehat{C}=i \gamma^{2} \gamma^{0}, \quad \bar{\Phi}=U(0,1)^{T}$, and $L_{j}=$ $\left(\nu_{L j} / \sqrt{2}, \ell_{L j}\right)^{T}$. We then quantitatively compute the high energy scattering $\nu_{L} \nu_{L} \rightarrow\left(\pi^{+}\right)^{\ell}\left(\pi^{-}\right)^{\ell}\left(\pi^{0}\right)^{n-2 \ell}$. In the actual calculation we use the real Majorana field $\chi=\left(\nu_{L}+\nu_{L}^{c}\right) / \sqrt{2}$ and compute the amplitude for three cases: (a) $n$ (even) $=2 \ell$; (b) $n$ (even) $>2 \ell$; (c) $n$ (odd) $\geqslant 3$. We derive the unitarity bound,

$$
E_{\nu}^{\star}=v\left[\left(\frac{v}{m_{\nu j}}\right)^{2} \frac{4 \pi}{\mathcal{R}_{\nu k}^{\max }}\right]^{\frac{1}{2(n-1)}},
$$


TABLE I: Summary of the strongest unitarity limit $E_{2 \rightarrow n}^{\star m i n}$ for each scattering $\xi_{1} \xi_{2} \rightarrow n V_{L}^{a}\left(\xi_{1,2}=V_{L}, f, \bar{f}, \nu_{L}\right)$ and the corresponding number of final state particles $n=n_{s}$, in comparison to the classic $2 \rightarrow 2$ limit $E_{2 \rightarrow 2}^{\star}$.

\begin{tabular}{c||c|cccccc|ccc|cc}
\hline \hline$\xi_{1} \xi_{2}$ & $V_{L}^{a_{1}} V_{L}^{a_{2}}$ & $t \bar{t}$ & $b \bar{b}$ & $c \bar{c}$ & $s \bar{s}$ & $d \bar{d}$ & $u \bar{u}$ & $\tau^{-} \tau^{+}$ & $\mu^{-} \mu^{+}$ & $e^{-} e^{+}$ & $\nu_{L} \nu_{L}$ \\
\hline \hline$M_{W}, m_{f, \nu}(\mathrm{GeV})$ & 80.4 & 178 & 4.85 & 1.65 & 0.105 & 0.006 & 0.003 & 1.777 & 0.106 & $5.11 \times 10^{-4}$ & $5 \times 10^{-11}$ \\
\hline$n_{s}$ & 2 & 2 & 4 & 6 & 8 & 10 & 10 & 6 & 8 & 12 & 22 \\
\hline$E_{2 \rightarrow n}^{\star(\min )}(\mathrm{TeV})$ & 1.2 & 3.49 & 23.4 & 30.8 & 52.1 & 77.4 & 83.6 & 33.9 & 56.3 & 107 & 158 \\
\hline \hline$E_{2 \rightarrow 2}^{\star}(\mathrm{TeV})$ & 1.2 & 3.49 & 128 & 377 & $6 \times 10^{3}$ & $10^{5}$ & $2 \times 10^{5}$ & 606 & $10^{4}$ & $2 \times 10^{6}$ & $1.1 \times 10^{13}$ \\
\hline \hline
\end{tabular}

where $\mathcal{R}_{\nu k}^{\max }(k=1,2,3)$ is a function of $n$ for three cases above [13]. From Fig. 4, we find the optimal limits,

$E_{\min }^{\star}=136,158,170 \mathrm{TeV}, \quad$ at $n=n_{s}=20,22,24$,

for typical inputs $m_{\nu j}=1.0,0.05,0.01 \mathrm{eV}$. This agrees with our estimate in Fig. 2 to within a factor of $\sim 1.7$.

\section{Conclusions}

In this work, we have systematically analyzed the unitarity limits from $2 \rightarrow n$ inelastic scattering; this provides a universal upper bound on the scales of mass generation for quarks, leptons and Majorana neutrinos. Table 1 summarizes our results in comparison with classic $2 \rightarrow 2$ limits [7, 10]. It shows that the scattering $f \bar{f} \rightarrow n V_{L}^{a}$ $(n \geqslant 2)$ does reveal a separate scale for fermion mass generation. Our new limits from $2 \rightarrow n$ scattering with $n>2$ establish new scales of mass generation for all light fermions including Majorana neutrinos, and are substantially stronger than the classic $2 \rightarrow 2$ limits. These new $2 \rightarrow n$ bounds vary within the range of $3-160 \mathrm{TeV}$ when the input fermion masses change from $m_{t}$ to $m_{\nu}$, as listed in Table 1 where the masses are pole-masses except that for $m_{u, d, s}$ the more precise $\overline{\mathrm{MS}}$ values are used 14$]$.

In particular, for Majorana neutrinos with typical masses $m_{\nu}=1.0-0.01 \mathrm{eV}$, the best upper limits on the scale of mass generation fall in the range $136-170 \mathrm{TeV}$ (with $n=20-24$ ). This is only a factor $\lesssim 7$ weaker than the lowest bound for light Dirac fermions despite their huge mass hierarchy $m_{\nu} / m_{f \neq t} \leqslant m_{\nu} / m_{b} \approx 2 \times\left(10^{-10}-\right.$ $\left.10^{-12}\right)$. Hence, these limits are insensitive to the variation of fermion masses. Such a strong non-decoupling feature for the scale of new physics associated with light fermion mass generation is essentially due to the chiral structure of fermion bare mass-terms, i.e., the fact that all left-handed SM fermions are weak-doublets but their right-handed chiral partners are weak singlets (or possibly absent for a radiatively generated Majorana neutrino mass). It is this feature that makes the coupling strength of fermions to multiple Goldstone bosons (or effectively, multiple longitudinal gauge bosons) proportional to the fermion mass, so the decoupling theorem [15] does not apply. For Majorana neutrino masses generated via the usual seesaw [3] or radiative [4] mechanism, this means that our new limits constrain the scale of the leptonic Higgs Yukawa interaction, which must invoke extra new fields (such as right-handed neutrinos or Zee-scalars or triplet Higgs) as needed for generating lepton number violation and ensuring renormalizability (although our new bounds do not directly constrain the masses of these new fields themselves which obey the decoupling theorem).

Finally, we have further estimated the $2 \rightarrow n$ unitarity limit on the EWSB scale via $V_{L}^{a_{1}} V_{L}^{a_{2}} \rightarrow n V_{L}^{a}$ $\left(\pi^{a_{1}} \pi^{a_{2}} \rightarrow n \pi^{a}\right)$ with $n \geqslant 2$, and find that the best limit remains $E_{W}^{\star} \simeq 1.2 \mathrm{TeV}$ with $n=n_{s}=2$. Also, the current study of $f \bar{f} \rightarrow n V_{L}^{a}\left(n \pi^{a}\right)$ scattering assumes $V_{L}^{a}\left(\pi^{a}\right)$ to be local field or remain local up to the limit $E_{f}^{\star}$. If $V_{L}^{a}\left(\pi^{a}\right)$ becomes composite much below $E_{f}^{\star}$, the pure fermion process $f \bar{f} \rightarrow(f \bar{f})^{n}$ may be useful for a model-independent analysis. A systematic expansion of this Letter is given in [13].

Acknowledgments We thank S. Willenbrock for valuable discussions. This work was supported by U.S. DOE under grant DE-FG03-93ER40757.
[1] Y. Ashie, et al., [SuperK-Kamiokande Collaboration], Phys. Rev. Lett. 93, 101801 (2004) hep-ex/0404034; O. Elgaroy and O. Lahav, New J. Phys. 7, 61 (2005).

[2] S. Weinberg, Phys. Rev. Lett. 43, 1566 (1979).

[3] P. Minkowski, Phys. Lett. B 67, 421 (1977); M. GellMann, P. Ramond, R. Slansky, in Proceedings of Workshop on Supergravity, eds. F. van Nieuwenhuizen and D. Freedman (Amsterdam, 1979), p.315; T. Yanagida, in Proceedings of Workshop on Unified Theories and Baryon Number in Universe, eds. O. Sawada and A. Sugamoto
(KEK, 1979), p.95; S. L. Glashow, in Quark and Leptons, eds. M. Levy et al. (Plenum, NY, 1980); R. N. Mohapatra and G. Senjanovic, Phys. Rev. Lett. 44, 912 (1980).

[4] A. Zee, Phys. Lett. B 93, 389 (1980); L. Wolfenstein, Nucl. Phys. B 175, 92 (1980); S. T. Petcov, Phys. Lett. B 115, 401 (1982).

[5] C. G. Callen, S. Coleman, J. Wess, and B. Zumino, Phys. Rev. 177, 2247 (1969).

[6] D. A. Dicus and V. S. Mathur, Phys. Rev. D 7, 3111 (1973); B. W. Lee, C. Quigg and H. B. Thacker, Phys. 
Rev. D 16, 1519 (1977); M. Veltman, Acta. Phys. Polon. B 8, 475 (1977).

[7] T. Appelquist and M. S. Chanowitz, Phys. Rev. Lett. 59, 2405 (1987).

[8] W. Marciano, G. Valencia, and S. Willenbrock, Phys. Rev. D 40, 1725 (1989).

[9] F. Maltoni, J. M. Niczyporuk, and S. Willenbrock, Phys. Rev. Lett. 86, 212 (2001) hep-ph/0006358.

[10] F. Maltoni, J. M. Niczyporuk, and S. Willenbrock, Phys. Rev. D 65, 033004 (2002) hep-ph/0106281.

[11] For a comprehensive review, H.-J. He, Y.-P. Kuang and
C.-P. Yuan, DESY-97-056 hep-ph/9704276.

[12] H.-J. He, Y.-P. Kuang and C.-P. Yuan, Phys. Rev. D 55, 3038 (1997); Phys. Lett. B 382, 149 (1996).

[13] D. A. Dicus and H.-J. He, Phys. Rev. D (2005), in Press, hep-ph/0409131; and also, H.-J. He and D. A. Dicus, presentation at DPF-2004, hep-ph/0411024

[14] Particle Data Group, Phys. Lett. B 592, 1 (2004) and http://pdg.lbl.gov.

[15] T. Appelquist and J. Carrazone, Phys. Rev. D 11, 2856 (1975). 